Part I

Supernovae: Individual 


\section{A Decade of Radio and X-ray Observations of SN 1993J}

Schuyler D. Van Dyk ${ }^{1}$, Kurt W. Weiler ${ }^{2}$, Richard A. Sramek ${ }^{3}$, Nino Panagia ${ }^{4}$, Christopher Stockdale ${ }^{5}$, Christina Lacey ${ }^{6}$, Marcos Montes ${ }^{2}$, and Michael Rupen ${ }^{3}$

1 IPAC/Caltech, Pasadena, CA, USA; vandyk@ipac.caltech.edu

2 NRL, Washington, DC, USA; Kurt.Weiler@nrl.navy.mil, Marcos.Montes@nrl.navy.mil

3 NRAO, Socorro, NM, USA; dsramek@aoc.nrao.edu, mrupen@aoc.nrao.edu

4 STScI, Baltimore, MD, USA \& Astrophys. Div., Space Sci. Dept., ESA; panagia@stsci.edu

5 Marquette Univ., Milwaukee, WI, USA;

Christopher.Stockdale@marquette.edu

${ }^{6} \mathrm{U}$. of South Carolina, Columbia, SC, USA; laceyosc.edu

Summary. We review ten years of radio continuum and X-ray monitoring of the Type IIb SN 1993J in M81. The supernova (SN) has been observed continuously, since only a few days after explosion, by our group with the Very Large Array at a number of radio frequencies, as well as by other groups. As a result, it is among the best-studied radio supernovae. The observed synchrotron radio emission is thought to arise from the interaction of the SN shock with the pre-SN wind-established circumstellar medium around the progenitor star. We describe the properties of the circumstellar interaction, based on the more fully-developed dataset, and compare this to our earlier characterization made in 1994. SN 1993J has also been a target of X-ray satellites, and we briefly discuss the nature of the X-ray emission and, together with the radio emission, describe the implications for the nature of the SN's progenitor.

\section{Introduction}

The radio emission from supernovae (SNe) serves as an excellent, and often the only, probe of the final stages of the evolution of the massive progenitor star. Radio SNe (RSNe) are characterized by nonthermal synchrotron emission, $S_{\nu}$, at very high brightness temperature $\left(T_{B}\right)$; "turn on" of the emission, first, at high radio frequencies and, subsequently, at lower frequencies; a power-law decline after maximum radio light at each frequency, with index $\beta$; and, transition from an optically thick spectral index, $\alpha$ (where $S_{\nu} \propto \nu^{\alpha}$ ). Type Ib/c RSNe turn on, reach maximum, and decline rapidly, e.g., at 6 $\mathrm{cm}$, within tens of days, while Type II (mostly II-linear; II-L) RSNe require several hundred days to rise to $6 \mathrm{~cm}$ maximum and then decline much more 
slowly than do the Type Ib/c RSNe. The Type II-narrow (IIn) RSNe evolve even more slowly, taking more than 1000 days to reach $6 \mathrm{~cm}$ maximum, before a slow decline over many years to decades. Observing most RSNe requires one to be a "sedentary gentle person of leisure," but this allows one to analyze these objects far more carefully and accurately as they evolve, compared to those who hastily and perfunctorily analyze various faster-evolving objects.

The radio emission has been interpreted via the Chevalier "mini-shell" model $[3,4]$, where the relativistic electrons and enhanced magnetic field necessary for synchrotron emission arise from the SN shock interacting with a relatively high-density circumstellar medium (CSM) which has been ionized and heated $\left(>10^{4}-10^{5} \mathrm{~K}\right)$ by the initial X-ray/UV flash. This CSM is presumed to have been established by a constant mass-loss $(\dot{M})$ rate, constant velocity $\left(w_{\text {wind }}\right)$ wind from a massive progenitor star or stellar system. The ionized CSM, then, is the source of free-free absorption of the synchrotron emission. A rapid rise in the observed radio flux density results from a decrease in absorption as the radio emitting region expands.

In this model, the spectral index $\alpha=(1-\gamma) / 2$, where $\gamma$ is the relativistic particle index, and the decline index $\beta=-(\gamma+5-6 m) / 2$, where the SN shock radius evolves with time as $R_{\text {shell }} \propto t^{m}$. The SN ejecta have density profile $\rho_{\text {ejecta }} \propto r^{-n}$, while $\rho_{\mathrm{CSM}}=\dot{M} /\left(4 \pi v_{\text {wind }} r^{-s}\right)$, such that $m=(n-3) /(n-s)$ (for a spherically-symmetric wind, $s=2$ ).

Chevalier [5] has said that "the expected time evolution of the physical parameters is not well understood and the predicted light curves are only plausible estimates of the evolution." Therefore, we have analyzed the observed data in terms of parameterized light curves. Originally, the parameterization was relatively simple and provided an adequate reproduction of the objects known at that time; see [25]:

$$
S(\mathrm{mJy})=K_{1}\left(\frac{\nu}{5 \mathrm{GHz}}\right)^{\alpha}\left(\frac{t-t_{0}}{1 \text { day }}\right)^{\beta} e^{-\tau_{\text {external }}}
$$

where

$$
\tau_{\text {external }}=K_{2}\left(\frac{\nu}{5 \mathrm{GHz}}\right)^{-2.1}\left(\frac{t-t_{0}}{1 \text { day }}\right)^{\delta} .
$$

$K_{1}$ and $K_{2}$ correspond formally to the flux density and uniform $\mathrm{f}$-f absorption (FFA) at $5 \mathrm{GHz}$ one day after the explosion date $t_{0}$. The absorption decline index $\delta=-3 m=\alpha-\beta-3$.

With the discovery of the RSN IIn 1986J, another layer of complexity was added to the parameterization, allowing for a clumpy or filamentary, mixed internal emission/absorption mechanism, which leads to a slower radio turnon; see [26]:

$$
S(\mathrm{mJy})=K_{1}\left(\frac{\nu}{5 \mathrm{GHz}}\right)^{\alpha}\left(\frac{t-t_{0}}{1 \text { day }}\right)^{\beta} e^{-\tau_{\text {external }}}\left(\frac{1-e^{-\tau_{\text {filament }}}}{\tau_{\text {filament }}}\right)
$$


where

$$
\tau_{\text {filament }}=\tau^{\prime}=K_{3}\left(\frac{\nu}{5 \mathrm{GHz}}\right)^{-2.1}\left(\frac{t-t_{0}}{1 \text { day }}\right)^{\delta^{\prime}},
$$

$K_{3}$ is the clumpy or filamentary absorption at $5 \mathrm{GHz}$ one day after explosion, and $\delta^{\prime}=5 \delta / 3$.

From the uniform, external FFA one can estimate the progenitor's massloss rate $[25,26,27]$ as

$$
\begin{aligned}
\frac{\dot{M}\left(\mathrm{M}_{\odot} \mathrm{yr}^{-1}\right)}{\left(w_{\text {wind }} / 10 \mathrm{~km} \mathrm{~s}^{-1}\right)}= & 3.0 \times 10^{-6} \tau_{\mathrm{FFA}}^{0.5} m^{-1.5}\left(\frac{v_{\mathrm{i}}}{10^{4} \mathrm{~km} \mathrm{~s}^{-1}}\right)^{1.5} \times \\
& \left(\frac{t_{\mathrm{i}}}{45 \text { days }}\right)^{1.5}\left(\frac{t}{t_{\mathrm{i}}}\right)^{1.5 m}\left(\frac{T}{10^{4} \mathrm{~K}}\right)^{0.68}
\end{aligned}
$$

where $t_{\mathrm{i}}$ is the measurement date of the initial ejecta velocity $v_{\mathrm{i}}$, and $T$ is the wind (CSM) temperature.

\section{Radio Emission from SN 1993J}

\subsection{Ancient History}

SN 1993J in M81 was monitored with the Ryle Telescope [18] and Very Large Array $\left(\mathrm{VLA}^{1} ;[24]\right)$, respectively, from just a few days after explosion up to the first 300 days. (We do not include discussion of the early mm data here.) Whereas Pooley \& Green [18] observed only at $2 \mathrm{~cm}$, Van Dyk et al. [24] provide data from 1.3 to $20 \mathrm{~cm}$ and found that the standard form of the Weiler et al. [26] parameterization did not provide an adequate fit to the data. Specifically, it became apparent, with $\delta=-1.99$ and $\delta^{\prime}=-2.02(\simeq 2)$, that the assumptions of $\delta=\alpha-\beta-3$ and $\delta^{\prime}=5 \delta / 3$ did not apply to SN 1993J.

With the results of the then-available VLBI data, that the SN was essentially in free expansion $[1,12]$, i.e., $m \simeq 1$, and with $\tau_{\text {FFA }} \propto \int n_{e}{ }^{2} d r \propto$ $r^{(-2 \eta+1)}, n_{e} \propto r^{-\eta}\left(\propto \rho_{\mathrm{CSM}}\right)$ and $\tau_{\mathrm{FFA}} \propto r^{-2}$, then $\eta=1.50$. That is, $\rho_{\mathrm{CSM}} \propto r^{-1.5}$, which is a shallower profile than for a spherically symmetric wind (see also [7]). Such a CSM profile could be explained by either a decreasing mass-loss rate, $\dot{M}$, or increasing wind speed, $w_{\text {wind }}$, prior to the progenitor's explosion. Such variation might also result in a clumpy wind.

1 The VLA telescope of the National Radio Astronomy Observatory is operated by Associated Universities, Inc. under a cooperative agreement with the National Science Foundation. 


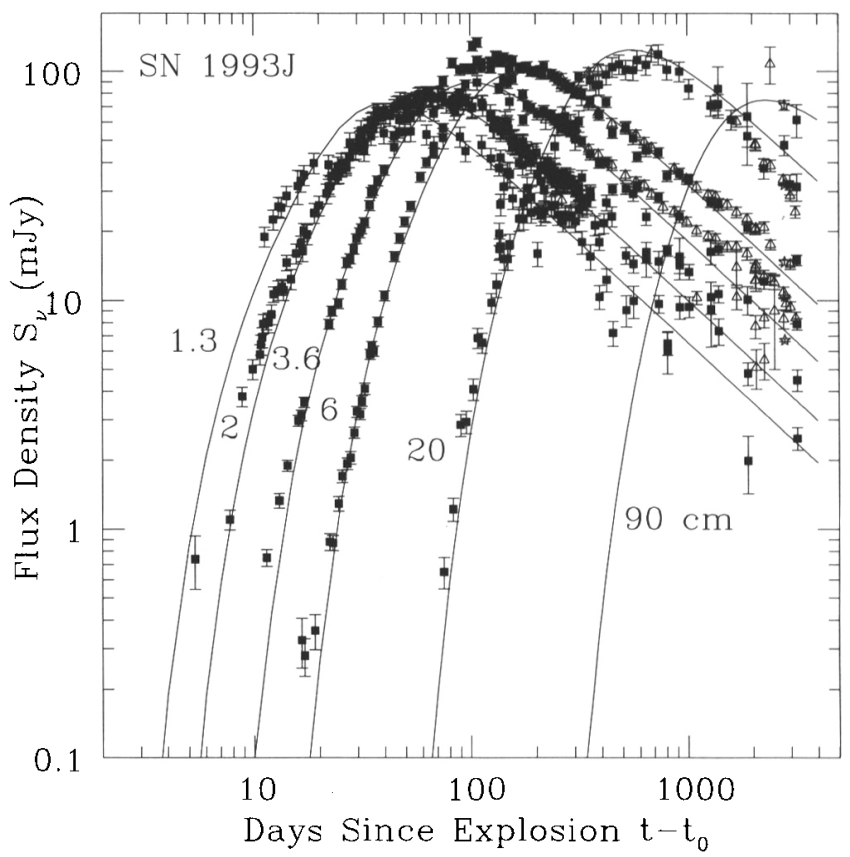

Fig. 1. Preliminary best-fit model to the SN 1993J VLA data. This model fit includes a clumpy, external absorbing medium and SSA.

\subsection{Alternate Views}

It has been proposed that synchrotron self-absorption (SSA) may play a role in some objects [5]. Fransson \& Bjornsson [8] and Chevalier [5] independently interpreted the SN 1993J data, assuming that the CSM profile was spherically symmetric (i.e., no shallow profile, no clumps) and that absorption is due to a combination of pure FFA and SSA. The model provided a good fit to the data. However, in this model the wind electron temperature, $T_{e}$, varies with radius, so that the FFA power law changes with radius and, therefore, with time. Although such an assumption is not physically unrealistic, an assumption of $T_{e}(r)$ has not been necessary to interpret any other RSN to date.

Similarly, Perez-Torres et al. [16] also model SN 1993J assuming pure FFA plus SSA and $T_{e}(r)$, but with $\rho_{\mathrm{CSM}} \propto r^{-1.66}$ (that the exponent is no longer assumed to be -1.5 is discussed below) and the assumption that the synchrotron emission is not optically thin. Again, their model provides a reasonable fit to the data. 


\subsection{Modern Times}

We have continued monitoring SN 1993J with the VLA at a number of frequencies, including $90 \mathrm{~cm}$, to $>4000$ days. The $\mathrm{SN}$ has also been observed at late times with the VLA by Bartel et al. [2] and Perez-Torres et al. [17]. SN 1993J continues to be, with the sole exception of SN 1987A, the beststudied RSN ever. However, the old fit [24] ceases to reproduce the data well, systematically overestimating the flux densities at late times. It has become necessary to refit our parameterization to the more complete dataset.

In recent times the parameterization has taken on additional complexity levels. A clumpy external medium plane-parallel absorption term has been included. Along with the mixed FFA/nonthermal emission internal to the interaction region, we also now include SSA in the formalism of $\tau_{\text {intennal }}$. Additionally, we also allow for the presence of a distant line-of-sight ionized medium, which results in a time-independent FFA component to $\tau_{\text {external }}$ and additional spectral turn-over at low frequencies (see [15]). The parameterization is now (see [27]):

$$
\begin{array}{r}
S(\mathrm{mJy})=K_{1}\left(\frac{\nu}{5 \mathrm{GHz}}\right)^{\alpha}\left(\frac{t-t_{0}}{1 \text { day }}\right)^{\beta} e^{-\tau_{\text {external }}}\left(\frac{1-e^{-\tau_{\mathrm{CSM}_{\mathrm{clumps}}}}}{\tau_{\mathrm{CSM}_{\mathrm{clumps}}}}\right) \times \\
\left(\frac{1-e^{-\tau_{\text {internal }}}}{\tau_{\text {internal }}}\right)
\end{array}
$$

where the new terms are

$$
\begin{gathered}
\tau_{\mathrm{CSM}_{\mathrm{clumps}}}=\tau^{\prime}=K_{3}\left(\frac{\nu}{5 \mathrm{GHz}}\right)^{-2.1}\left(\frac{t-t_{0}}{1 \text { day }}\right)^{\delta^{\prime}} \\
\tau_{\text {internal } \mathrm{SSA}}=K_{5}\left(\frac{\nu}{5 \mathrm{GHz}}\right)^{\alpha-2.5}\left(\frac{t-t_{0}}{1 \text { day }}\right)^{\delta^{\prime \prime}}
\end{gathered}
$$

and

$$
\tau_{\text {distant }}=\tau^{\prime \prime}=K_{4}\left(\frac{\nu}{5 \mathrm{GHz}}\right)^{-2.1}
$$

and where $K_{3}$ is now the clumpy external absorption, $K_{5}$ is the internal SSA, and $K_{4}$ is the external time-independent FFA, all at $5 \mathrm{GHz}$ one day after explosion. (Of course, in addition to clumps or filaments, the CSM may be structured with significant density irregularities, such as rings, disks, or shells.)

In Fig. 1 we show a preliminary best fit to the more complete data, including both an external clumpy medium and SSA, with $\chi^{2} /$ dof $=5.48$. The simpler assumption of wind $T_{e}=$ const is adopted. In Table 1 (column 2) we list the best-fit model parameters. The main point emphasized here is that, 


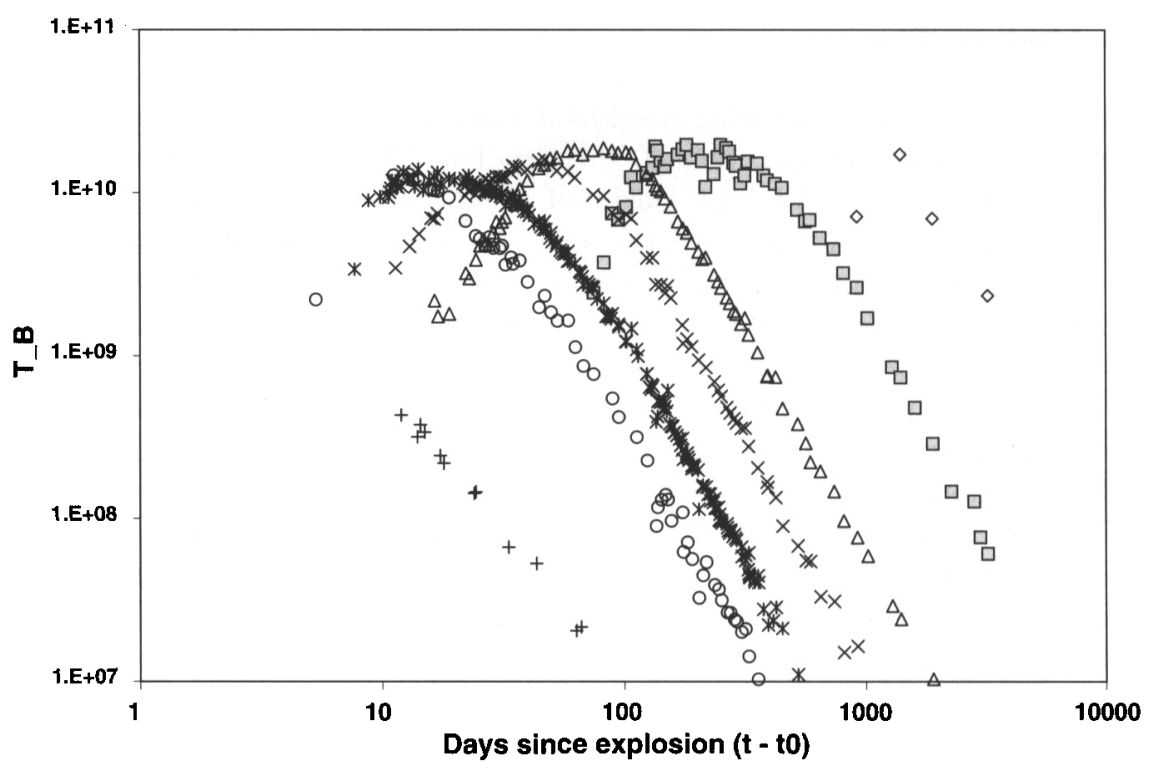

Fig. 2. Brightness temperature of SN 1993J based on VLA data, assuming $v_{\text {exp }}=$ $10^{4} \mathrm{~km} \mathrm{~s}^{-1}$.

for SN 1993J, SSA is not important, except for very early times, and SN $1993 \mathrm{~J}$ bears no relationship at all to a sGRB. To further illustrate this, in Fig. 2 we show $T_{B}$ as a function of time, based on the data at all frequencies and assuming $v_{\exp }=10^{4} \mathrm{~km} \mathrm{~s}^{-1}$. One can see that SN 1993J comes nowhere near experiencing any "inverse Compton catastrophe," and $T_{B}<10^{11} \mathrm{~K}$ is well under even the equipartition $T_{B}$ [19] that presumably sets a hard limit. SSA is simply not essential for SN 1993J. Based on this best-fit model, in Fig. 3 we show the absorption with time, and one can readily see that the SSA declines far more rapidly than the FFA and generally does not contribute substantially to the overall absorption.

Therefore we also can apply a preliminary best-fit model $\left(\chi^{2} /\right.$ dof $\left.=5.69\right)$ to the data which leaves out SSA completely, but instead includes only FFA from the CSM (both uniform and clumpy). We show the fit in Fig. 4. As one can see, both models fit equally well (due to the relatively negligible SSA); both models also do not quite fit the peaks of the light curves well. In Table 1 (column 3) we list the best-fit model parameters. What can be seen is what was found in the past [24]: $\delta \simeq \delta^{\prime} \simeq 2$. Using the more recent VLBI results, i.e., $m=0.86[2,13]$, following similar arguments as above, one finds $s=1.66$, i.e., $s \neq 2$ (see also [14]). The main conclusions from the past $[7,24]$ still apply for the more complete dataset. 


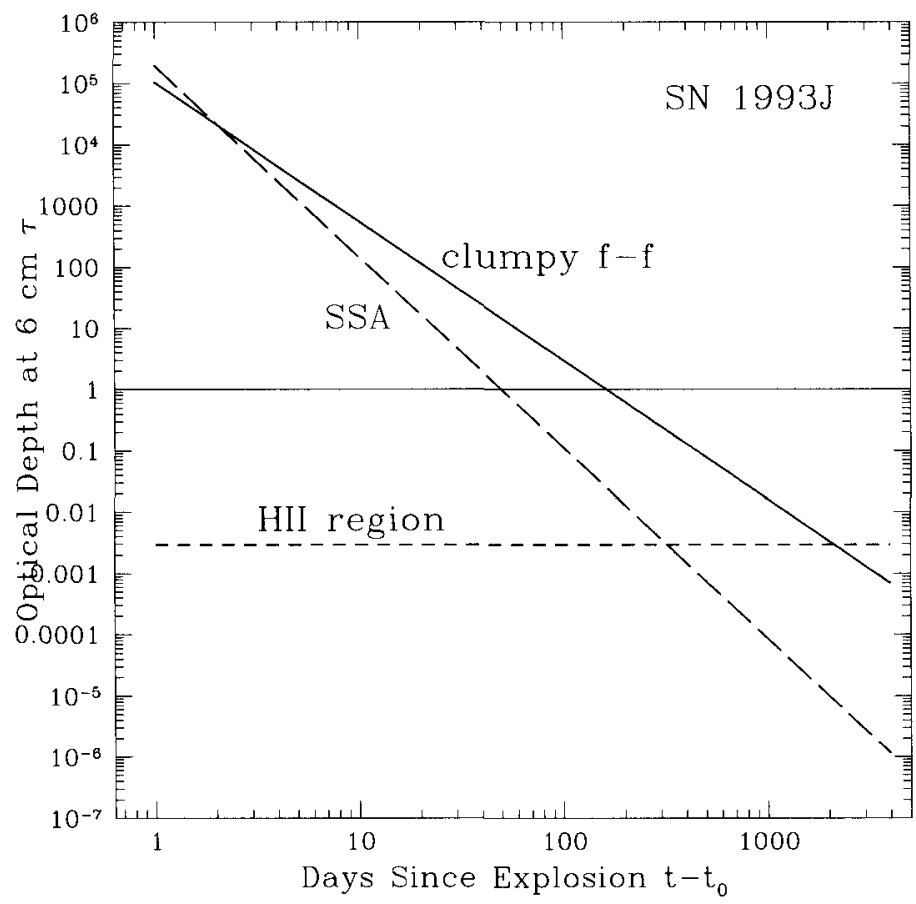

Fig. 3. Evolution of absorption of synchrotron radio emission from SN 1993J, based on a preliminary best-fit model. Shown are the clumpy FFA, SSA, and timeindependent FFA from a distant line-of-sight HII region.

Table 1. Best-Fit Parameters

\begin{tabular}{ccc}
\hline Parameter & Value(1) & Value(2) \\
\hline$K_{1}$ & $1.39 \times 10^{4}$ & $1.36 \times 10^{4}$ \\
$\alpha$ & -1.04 & -1.05 \\
$\beta$ & -0.88 & -0.88 \\
$K_{2}$ & $\ldots$ & $9.14 \times 10^{2}$ \\
$\delta$ & $\ldots$ & -1.88 \\
$K_{3}$ & $9.98 \times 10^{4}$ & $8.33 \times 10^{4}$ \\
$\delta^{\prime}$ & -2.27 & -2.26 \\
$K_{4}$ & $2.76 \times 10^{-3}$ & $2.76 \times 10^{-3}$ \\
$K_{5}$ & $1.78 \times 10^{5}$ & $\cdots$ \\
$\delta^{\prime \prime}$ & -3.12 & $\cdots$ \\
\hline
\end{tabular}

\subsection{A New Wrinkle?}

As can be seen in both Figs. 1 and $4, \alpha$ appears to be changing, from $\approx-1$ to $\approx-0.6$ or -0.7 (see $[2,17]$ ); at late times the overall model, with $\alpha \simeq-1$, underestimates the flux densities. We are investigating this change in our 


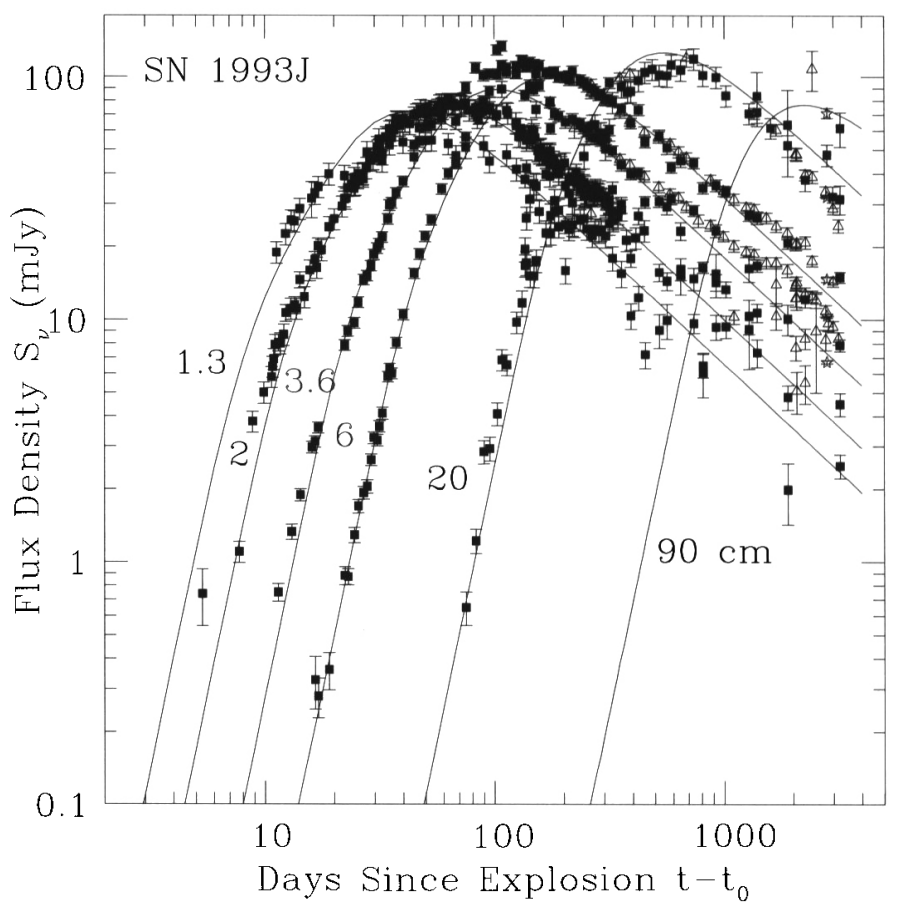

Fig. 4. Preliminary best-fit model to the SN 1993J VLA data. This model fit includes both a clumpy and uniform external absorbing medium, but no SSA.

continued VLA monitoring. The change could be due to increased adiabatic cooling and Coulomb losses [17] or the shock expanding into a region of CSM with a steeper density profile [2].

\section{X-ray Emission from SN 1993J}

Space does not afford a full discussion of X-rays from SN 1993J. Fortunately, this topic is discussed in more detail by Immler, Zimmermann, and Pooley in this volume. The SN was observed early using ROSAT at $0.1-2.4 \mathrm{keV}[28,29]$, $A S C A$ at $1-10 \mathrm{keV}[10,22]$, and CGRO/OSSE at 50-150 keV [11], and at late times using Chandra at 0.3-8.0 keV [21] and XMM [30]. The main result is that X-rays were hard early and have become progressively softer. (See, e.g., the hardness index from the ASCA data [23].) This can best be explained by the hard X-rays emerging from the adiabatically expanding forward shock at $\sim 10^{9} \mathrm{~K}$, and the softer X-rays from the radiative reverse shock at $\sim 10^{7} \mathrm{~K}$. At early times a cool shell, at $<10^{4} \mathrm{~K}$ at the shock contact discontinuity, absorbed most of the soft X-rays from within; see $[6,7]$. From the linear decline of the ROSAT light curve Immler et al. [9] also find that $s=-1.63$, 
confirming the earlier radio results [24] (see also [20]; although Swartz et al. [21] find a break in the light curve) and conclude that either $w_{\text {wind }}$ was increasing and/or $\dot{M}$ was decreasing, as the progenitor transitioned from a red to blue supergiant prior to explosion.

The study of this fascinating $\mathrm{SN}$ at both radio and $\mathrm{X}$-ray wavelengths continues.

Acknowledgement. We thank C. Williams for assistance in the VLA data analysis. KWW wishes to thank the Office of Naval Research (ONR) for the 6.1 funding supporting this research. Additional information and data on RSNe can be found on http://rsd-www.nrl.navy.mil/7213/weiler/ and linked pages.

\section{References}

1. N. Bartel et al. : Nature 368, 610 (1994)

2. N. Bartel et al. : Astrophys. J. 581, 404 (2002)

3. R.A. Chevalier: Astrophys. J. 259, 302 (1982)

4. R.A. Chevalier: Astrophys. J. Lett. 258, L63 (1984)

5. R.A. Chevalier: Astrophys. J. 499, 810 (1998)

6. R.A. Chevalier, C. Fransson: Astrophys. J. 420, 268 (1994)

7. C. Fransson, P. Lundqvist, R.A. Chevalier: Astrophys. J. 461, 993 (1996)

8. C. Fransson, C.-I. Björnsson: Astrophys. J. 509, 861 (1998)

9. S. Immler, B. Aschenbach, Q.D. Wang: Astrophys. J. Lett. 561, L107 (2001)

10. Y. Kohmura et al. : Pub. Astron. Soc. Japan 46, L157 (1994)

11. M.D. Leising et al. : Astrophys. J. Lett. 431, L95 (1994)

12. J. Marcaide et al. : Astrophys. J. Lett. 424, L25 (1994)

13. J.M. Marcaide et al. : Astrophys. J. Lett. 486, L31 (1997)

14. A.J. Mioduszewski, V.V. Dwarkadas, L. Ball: Astrophys. J. 562, 869 (2001)

15. M.J. Montes, K.W. Weiler, N. Panagia: Astrophys. J. 488, 792 (1997)

16. M.A. Pérez-Torres, A. Alberdi, J.M. Marcaide: Astron. Astrophys. 374, 997 (2001)

17. M.A. Pérez-Torres, A. Alberdi, J.M. Marcaide: Astron. Astrophys. 394, $71(2002)$

18. G.G. Pooley, D.A. Green: Mon. Not. R. Astron. Soc. 264, 17P (1993)

19. A.C.S. Readhead: Astrophys. J. 426, 51 (1994)

20. T. Suzuki, K. Nomoto: Astrophys. J. 455, 658 (1995)

21. D.A. Swartz et al. : Astrophys. J. 144, 213 (2003)

22. Y. Tanaka: IAUC 5753 (1993)

23. S. Uno et al. : Astrophys. J. 565, 419 (2002)

24. S.D. Van Dyk, K.W. Weiler, R.A. Sramek, M.P. Rupen, N. Panagia: Astrophys. J. Lett. 432, L115 (1994)

25. K.W. Weiler et al. : Astrophys. J. 301, 790 (1986)

26. K.W. Weiler, N. Panagia, R.A. Sramek: Astrophys. J. 364, 611 (1990)

27. K.W. Weiler, N. Panagia, M.J. Montes, R.A. Sramek: Ann. Rev. Astron. Astrophys. 40, 387 (2002)

28. H.-U. Zimmermann, W. Lewin, E. Magnier, et al. : IAUC 5748 (1993)

29. H.-U. Zimmermann, W. Lewin, P. Predehl, et al. : Nature 367, 621 (1994)

30. H.-U. Zimmermann, B. Aschenbach: Astron. Astrophys. 406, 969 (2003) 